\title{
A computer-based programmable tachistoscope for nonprogrammers
}

\author{
DENNIS NORRIS \\ Medical Research Council Applied Psychology Unit, Cambridge, England
}

\begin{abstract}
A set of programs for on-line control of reaction time experiments is described. These programs allow a wide range of experiments to be coded in a simple form that eliminates the need for any knowledge of programming.
\end{abstract}

Assembling the hardware required to run computercontrolled psychological experiments is rapidly becoming both cheaper and easier. However, once one has the hardware, the process of preparing an experiment often leaves nonprogrammers longing for the good old days of the tachistoscope and stopwatch.

In a comprehensive review of computer-based tachistoscope systems, Green and Schwartz (1978) described three types of systems designed to simplify the process of preparing software for psychological experiments. The most popular approach has been to provide the user with a set of callable subroutines that perform basic operations such as timing, stimulus display, and response logging. A good example of such a system is the TSP package described by Schwartz (1978). This package provides a flexible set of functions that can be combined to create programs to run a wide range of psychological experiments. The TSP package does not eliminate the need for programming altogether, but it does make the task very much easier. However, most programming languages still provide the inexperienced user with plenty of opportunity for making mistakes. A second approach is to devise a special-purpose language for experimental control. However, once again the naive user has to master at least the fundamentals of programming. The third approach is to construct a filedriven system in which the user has to prepare a data file that specifies the details of the experiment. However, in a file-driven system such as Mewhort's (1978) DIS system, the user has to explicitly supply all of the timing information for every display in the experiment. In principle, the user does not need to know how to program to use this system. However, to make effective use of the system, one really needs to be able to write programs that will generate the file containing all of the control information automatically. With the DIS system, one does not have to be able to program, but it helps.

The author's mailing address is: Medical Research Council Applied Psychology Unit, 15 Chaucer Road, Cambridge CB2 2EF, England.
The present paper describes a file-driven system designed to overcome the limitations of systems such as DIS, and to eliminate the need for the user to have any knowledge of programming. The TSCOP system consists of a set of programs for preparing and running a wide range of reaction time experiments. These programs provide a very simple means of coding instructions for stimulus presentation and response timing for any experiment involving presentation of text that might once have been run on a tachistoscope. The amount of timing and control information that the user must supply is kept to an absolute minimum. In the simplest case, experiments can be prepared in little more time than is required to type the materials.

To date, two versions of the system have been implemented, one on a PDP-11/03 running RT-11 and the other on a PDP-11/40 running UNIX. The UNIX-based version of the system operates by downloading crosscompiled programs to Motorola M6809 microprocessors that perform all real-time control functions. Both systems use Televideo 912 or 920 terminals for stimulus display. The terminals are modified to allow cursor blanking and frame-scan synchronization. The RT-11 system is written entirely in Whitesmith's $C$ and should therefore be relatively simple to implement on many stand-alone micros. The system will run in about $32 \mathrm{~K}$, but a minimum of about $54 \mathrm{~K}$ is required to run the Whitesmith's compiler.

The basic system consists of the following three essential programs:

1. TSCOP-program for translating coded specification of timing parameters and stimuli into a data structure for input to 2 .

2. EXP-experimental control program. This program steps its way through data structure created by TSCOP to display stimuli and record responses.

3. MSORT-data-sorting program. Prints reaction times and response identifiers for individual trials or groups of trials. Computes condition means and sizes of interactions, and formats data for input to a statistical package, such as ALICE.

In addition, there are two further programs designed to facilitate construction of stimulus lists: 
4. MRAND-program to reorder or randomize stimulus lists for input to TSCOP.

5. MORD-program to generate pseudorandom sequencies to drive MRAND.

Wherever possible, all intermediate files are in ASCII so that, if programs do not do exactly as required, the user can alter the files with a conventional text editor. The use of ASCII files also allows the user to keep track of exactly what each program does.

The combination of the programs TSCOP and EXP simulates an $n$-field programmable tachistoscope. The timing characteristics of an experiment are specified by a list of parameters at the head of the file containing the stimuli. The timing characteristics can be altered as often as required during an experiment by introducing a new set of parameters into the stimulus file. Each set of timing parameters is entered on a new line headed with "*" and terminated by a colon or semicolon. Comments are permitted before the first "*." The first number in each parameter sequence specifies the duration in milliseconds of the blank field to precede the first display, the second number specifies the duration of the first display, the third, the duration of the next blank field, etc. The programs continue to cycle through each set of parameters until a new set is encountered or until the experiment is completed. The timing parameters can be followed by any number of displays (words, sentences, paragraphs, etc.), each terminated by a lefthand parenthesis. The parenthesis is optionally followed by an integer that can be used to determine the order in which data are printed. These integers are generated automatically when MRAND is used.

There are five main classes of parameters:

1. Positive numbers. The field remains on for the time specified or until the subject responds, whichever is first.

2. Negative numbers. The field remains on for the time specified, regardless of the subject's response.

3. Unity. When the time specified is $1 \mathrm{msec}$, the field remains on until the subject responds.

4. Minus one. A time of -1 causes the field to remain on until there is an input on a specially reserved input line. This facility is used to permit synchronization with external events, such as timing signals on tape recorders.

\section{Zero. The field is not displayed.}

Each parameter can be preceded by one or all of the following control characters:

$\mathrm{h}-$ The field is displayed at half intensity.

$\mathrm{n}$-There is no centering. Displays are centered horizontally as default.

$\mathrm{s}-$ The response time and identification for that frame are stored.

$x$-The next four digits are to be interpreted as a pair of two-digit $x$ - and $y$-coordinates.

Figure 1 shows how one might code a simple lexical decision experiment. The subject in this experiment is to be presented with two words on each trial. The first word is a prime to which no response is required, and

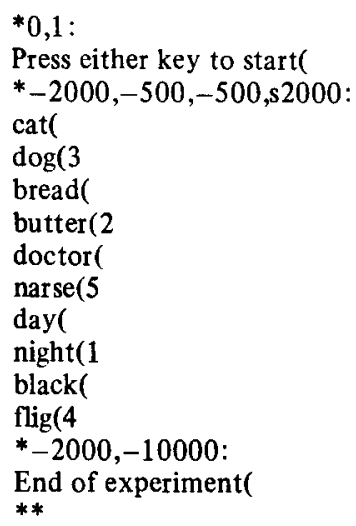

Figure 1.

the second is the decision word to which a response must be made. The first set of parameters indicates that the first stimulus is to be displayed without any preceding blank field and is to remain on until the subject presses a button. The second set of parameters specifies the timing sequence used for the main part of the experiment. There will be a blank field for $2,000 \mathrm{msec}$, after which the prime word will be displayed for $500 \mathrm{msec}$. There will then be a further blank field of $500 \mathrm{msec}$, followed by the decision word. The decision word will remain on until $2,000 \mathrm{msec}$ has elapsed or until the subject responds, whichever occurs first. If the subject responds, the response time will be stored along with an indication of which response key was pressed. Responses elsewhere in the timing sequence will be ignored. This timing sequence will be repeated for all pairs of stimuli until the program encounters a new set of parameters. The final set of parameters indicates that there will be a 2,000-msec blank field following the final word, after which the "End of experiment" message will remain on for $10 \mathrm{sec}$. In this example, the response to "night" would be printed out first, the response to "butter" second, and the response to "dog" third, etc.

At the most simple level, these programs do little more than simulate a tachistoscope and $\log$ responses. The user types in the specification of the timing cycle and then a list of stimuli in the order in which they are to be presented. However, the programs MRAND and MORD were designed to facilitate the construction of more complex experiments. Preparation of a typical experiment would involve creating a master file containing a list of all stimuli to be used in the experiment. MRAND and MORD can then be used to extract arbitrary subsets of the stimuli and to randomize trials either within individual subsets or across groups of subsets to form new lists. The programs keep a record of their operations, which MSORT can use to sort data. Reaction times are then printed out in the order in which the corresponding trials appeared in the master file. MSORT will screen the data for incorrect responses and responses outside a specified time window, and will 
compute condition means and perform simple arithmetic on condition means.

The main function of TSCOP is to create a data structure in which all timing and display information is represented explicitly. This means that EXP is a relatively simple program. All it has to do is to step through the data structure and $\log$ responses. Therefore, even with fairly large experiments, both display information and responses can fit into $32 \mathrm{~K}$. In the UNIX-based version of the system, real-time control is delegated to microprocessors with $32 \mathrm{~K}$ RAM, but with no disk or tape storage of their own. The microprocessors each have two serial lines, one connected to UNIX and the other to the experimental terminal. In normal use, the microprocessor is transparent to the user, and the terminal behaves as if it were connected directly to UNIX. To run an experiment, programs are downloaded from UNIX to the microprocessor. The experiment can then be run at any time, even if UNIX crashes. At the end of the experiment, data can be sent back to UNIX and stored on the UNIX file system.

This system, therefore, combines the advantages of a flexible multiuser operating system with the advantages of a dedicated microprocessor for experimental control. All of the work involved in preparing an experiment can be carried out at any terminal on the UNIX system, thus allowing the user to take full advantage of UNIX utilities. For example, experiments involving presentation of text can use nroff to format stimuli.

The UNIX-based version of this system has now been in operation at Sussex University for over 2 years. For the completely naive user, the most difficult part of learning to use this system usually has been learning to use a text editor. The rest of the system is easier to use than the tachistoscopes it replaced.

\section{REFERENCES}

Green, B. F., \& Schwartz, S. P. (1978). Comparative evaluation of computer-based tachistoscopes. Behavior Research Methods \& Instrumentation, 10, 789-795.

MEwhоRT, D. J. K. (1978). An n-channel tachistoscope algorithm. Behavior Research Methods \& Instrumentation, 10, 756-760.

Schw ARTz, S. P. (1978). Tachistoscope simulation package. Behavior Research Methods \& Instrumentation, 10, 773-778.

(Manuscript received September 9, 1983; revision accepted for publication February 17, 1984.) 